\title{
Adoptive immunotherapy with liver allograft- derived lymphocytes induces anti-HCV activity after liver transplantation in humans and humanized mice
}

\author{
Masahiro Ohira, 1,2 Kohei Ishiyama,, 1,2 Yuka Tanaka,1,2 Marlen Doskali,1,2 Yuka Igarashi,1,2 \\ Hirotaka Tashiro, 1,2 Nobuhiko Hiraga, 2,3 Michio Imamura, ${ }^{2,3}$ Naoya Sakamoto, ${ }^{4}$ \\ Toshimasa Asahara, 1,2 Kazuaki Chayama,2,3 and Hideki Ohdan1,2
}

1Department of Surgery, Division of Frontier Medical Science, Programs for Biomedical Research, Graduate School of Biomedical Sciences, 2Liver Research Project Center, and ${ }^{3}$ Department of Medicine and Molecular Science, Division of Frontier Medical Science, Programs for Biomedical Research Graduate School of Biomedical Sciences, Hiroshima University, Minami-ku, Hiroshima, Japan. ${ }^{4}$ Department of Gastroenterology and Hepatology, Tokyo Medical and Dental University, Bunkyo-ku, Tokyo, Japan.

\begin{abstract}
After liver transplantation in HCV-infected patients, the virus load inevitably exceeds pre-transplantation levels. This phenomenon reflects suppression of the host-effector immune responses that control HCV replication by the immunosuppressive drugs used to prevent rejection of the transplanted liver. Here, we describe an adoptive immunotherapy approach, using lymphocytes extracted from liver allograft perfusate (termed herein liver allograft-derived lymphocytes), which includes an abundance of NK/NKT cells that mounted an anti-HCV response in $\mathrm{HCV}$-infected liver transplantation recipients, despite the immunosuppressive environment. This therapy involved intravenously injecting patients 3 days after liver transplantation with liver allograft-derived lymphocytes treated with IL-2 and the CD3-specific mAb OKT3. During the first month after liver transplantation, the HCV RNA titers in the sera of recipients who received immunotherapy were markedly lower than those in the sera of recipients who did not receive immunotherapy. We further explored these observations in human hepatocyte-chimeric mice, in which mouse hepatocytes were replaced by human hepatocytes. These mice unfailingly developed $\mathrm{HCV}$ infections after inoculation with $\mathrm{HCV}$-infected human serum. However, injection of human liver-derived lymphocytes treated with IL-2/OKT3 completely prevented HCV infection. Furthermore, an in vitro study using genomic $\mathrm{HCV}$ replicon-containing hepatic cells revealed that IFN- $\gamma$-secreting cells played a pivotal role in such anti-HCV responses. Thus, our study presents what we believe to be a novel paradigm for the inhibition of $\mathrm{HCV}$ replication in $\mathrm{HCV}$-infected liver transplantation recipients.
\end{abstract}

\section{Introduction}

Liver failure and hepatocellular carcinoma (HCC) due to chronic hepatitis $\mathrm{C}$ infection are the most common indications for liver transplantation (LT), and the incidences of both have been projected to increase further in the future. Recurrent $\mathrm{HCV}$ infection of the allograft is universal, occurs immediately after LT, and is associated with accelerated progression to cirrhosis, graft loss, and death $(1,2)$. This reflects the suppression of those host-effector immune responses that usually control HCV replication, suggesting that the immunosuppressive environment may play a major role in the rapid progression of recurrent $\mathrm{HCV}$ infection after LT $(3,4)$. Further, the immunosuppressive condition described above is considered to increase the incidence of cancer recurrence after LT in HCC patients. We recently proposed the novel strategy of adjuvant immunotherapy for preventing the recurrence of HCC after LT; this immunotherapy involves intravenously injecting LT recipients with activated liver allograft-derived NK cells $(5,6)$. Since the immunosuppressive regimen currently used after LT reduces the adaptive immune components but effectively maintains the innate components of cellular immunity (7-9), the augmenta-

Conflict of interest: The authors have declared that no conflict of interest exists. Citation for this article: J. Clin. Invest. 119:3226-3235 (2009). doi:10.1172/JCI38374. tion of the NK cell response, which is thought to play a pivotal role in innate immunity, may be a promising immunotherapeutic approach (6). We confirmed that the IL-2/anti-CD3 mAb-treated (IL-2/OKT3-treated) liver allograft-derived NK cells expressed a significantly high level of the tumor necrosis factor-related apoptosis-inducing ligand (TRAIL), which is a critical molecule for tumor cell killing. Further, these cells showed high cytotoxicity against HCC cells, with no such effect on normal cells (5). After obtaining approval from the ethical committee of our institute, we successfully administered adoptive immunotherapy with IL-2/ OKT3-treated liver lymphocytes to liver cirrhosis patients with HCC in a phase I trial. Although the long-term benefits of this approach with regard to the control of HCC recurrence after LT remain to be elucidated, this trial provided a unique opportunity to study whether the adoptive administration of IL-2/OKT3-treated liver lymphocytes could also mount an anti-HCV response in HCVinfected LT recipients.

Previous studies have highlighted the important roles of innate lymphocytes in developing immunity against hepatotropic viruses, including $\operatorname{HCV}(10,11)$. In this regard, it is known that patients with chronic HCV infection show diminished NK and NKT cell responses (12-14). In the case of an LT, it has recently been reported that the host $\mathrm{CD}^{2} 6^{+}$innate lymphocyte population, 


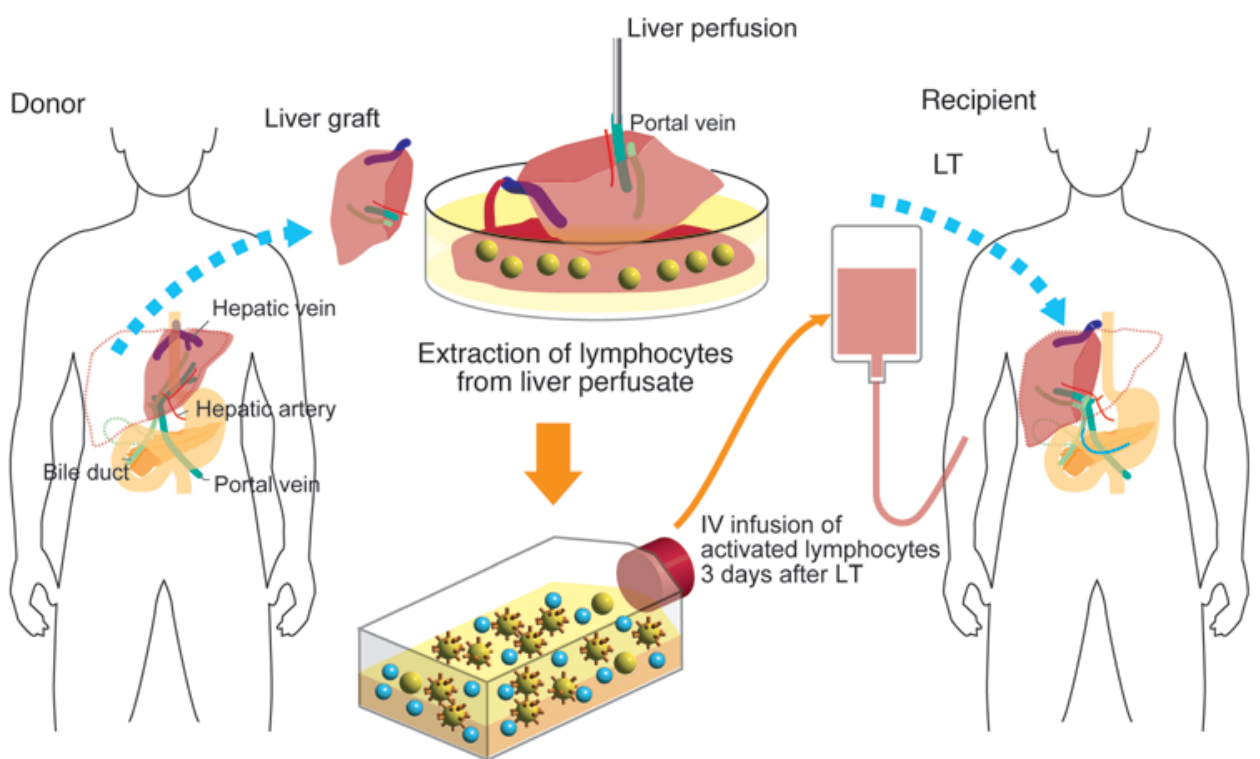

\section{Figure 1}

Schematic outline of adoptive immunotherapy with lymphocytes extracted from liver allograft perfusate. The therapy involved giving an intravenous injection of IL-2/OKT3-treated liver lymphocytes to $L T$ recipients. The lymphocytes were extracted from the donor liver graft perfusate. After 3 days of culture with IL-2 (100 $\mathrm{JRU} / \mathrm{ml}$ ), the activated liver NK cellenriched lymphocytes were administered to the $L T$ recipients through venous circulation. OKT3 $(1 \mu \mathrm{g} / \mathrm{ml})$ was added to the culture medium 1 day before this administration in order to prevent GVHD.

Stimulate lymphocytes with IL-2 for 3 days Add OKT3 in order to deplete T cells

consisting of NK and NKT cells, is appreciably associated with the severity of $\mathrm{HCV}$ recurrence after LT (15). These insights into the immunopathogenesis of $\mathrm{HCV}$ recurrence indicate that the innate immune components mentioned above are potential targets for therapeutic manipulation. In this study, we have demonstrated for the first time to our knowledge that adoptive immunotherapy with IL-2/OKT3-treated liver lymphocytes, including abundant NK and NKT cells, shows anti-HCV activity after LT, even in an immunosuppressive environment.

\section{Results}

Adoptive transfer of IL-2/OKT3-treated liver lymphocytes. The human liver contains a significant number of resident lymphocytes. These cells include abundant CD56 $6^{+} \mathrm{NK}$ and NKT cells, many of which differ phenotypically and functionally from the circulating cells $(14,16)$. In our previous study, we performed ex vivo perfusion of the liver through the portal vein, which was necessary in order to flush blood from the liver graft before implantation. Liver-resident lymphocytes were then extracted from the perfusates (number of lymphocytes extracted from normal liver perfusates, $0.5 \pm 0.1$ cells per gram of liver weight; $n=14)(5)$. Proportions of CD56 $6^{+} \mathrm{CD} 3^{-}$ $\mathrm{NK}$ cells and $\mathrm{CD} 56^{+} \mathrm{CD}^{+} \mathrm{NKT}$ cells among the lymphocytes extracted from the liver perfusates (NK cells, $46.4 \% \pm 4.2 \%$; NKT cells, $17.2 \% \pm 2.3 \% ; n=14)$ were significantly $(P<0.05)$ higher than those among the lymphocytes derived from the peripheral blood of the same donors (NK cells, $21.9 \% \pm 3.7 \%$; NKT cells, $3.8 \% \pm 0.9 \%$; $n=14$ ). Extensive preclinical studies have shown that liver allograft-derived resident NK cells mediate remarkably higher cytotoxic activity against HCC cells than do peripheral blood NK cells (5). On this basis, we undertook a clinical trial of adjuvant immunotherapy with IL-2/OKT3-treated liver lymphocytes for preventing the recurrence of HCC after LT in 14 recipients with HCC (Figure 1 and Tables 1 and 2). The therapy involved administering a single intravenous injection of IL-2/OKT3-treated liver lymphocytes to recipients 3 days after LT $\left(2-5 \times 10^{8}\right.$ cells injected per subject). In order to prevent graft-versus-host disease (GVHD), i.e., to inactivate $\mathrm{CD}^{+}$alloreactive $\mathrm{T}$ cells, we added an anti-CD3 $\mathrm{mAb}, \mathrm{OKT} 3$, to the culture medium a day before the inoculation. During the follow-up period (mean, 23.4 months; range, 10.7-32.9 months), neither any remarkable adverse effects nor rejection episodes occurred. All 14 subjects who received the immunotherapy were alive without recurrence of HCC after LT (including 5 patients with HCC exceeding the Milan criteria; ref. 17). At our institute, the survival rate and recurrence rate of historical control patients with HCC exceeding the Milan criteria were $78 \%$ (30 of 37) and $10.8 \%$ ( 4 of 37 ), respectively. The lymphocytes in the peripheral blood of LT recipients who received immunotherapy in the early postoperative period showed significantly enhanced cytotoxicity against an HCC cell line (HepG2) as compared with those in the peripheral blood of LT recipients who did not receive the therapy in the same period (Figure 2A). Although the gross proportions of NK/NKT cells in the peripheral blood of patients treated with immunotherapy did not differ from those in the peripheral blood of untreated patients, the proportions of TRAIL ${ }^{+} \mathrm{NK}$ cells significantly increased after immunotherapy in the peripheral blood of the former patients. This increase in the TRAIL ${ }^{+} \mathrm{NK}$ cells in the peripheral blood lymphocytes was not observed in untreated patients (Figure 2B). Furthermore, there was a significant correlation between the frequency of $\mathrm{TRAIL}^{+} \mathrm{NK}$ cells in the peripheral blood lymphocytes and the NK cytolytic activity of the peripheral blood lymphocytes at 7 days after LT (Spearman rank-order correlation coefficient $=0.54, P=0.01$; Figure $2 \mathrm{C}$ ), indicating the anti$\mathrm{HCC}$ effect of adoptively injected $\mathrm{TRAIL}^{+} \mathrm{NK}$ cells. It would be pertinent to conduct additional clinical trials of this immunotherapy for preventing HCC recurrence after LT.

Anti-HCV activity after adoptive immunotherapy. Of the 14 LT recipients who received the immunotherapy, 7 had chronic HCV infection. During the period of this trial, 5 other HCV-infected LT recipients who did not agree to receive immunotherapy served as controls; the background of the controls, including HCV genotype, age, and immunosuppressive therapy, was similar to that of the immunotherapy recipients (Table 3). It has been reported 


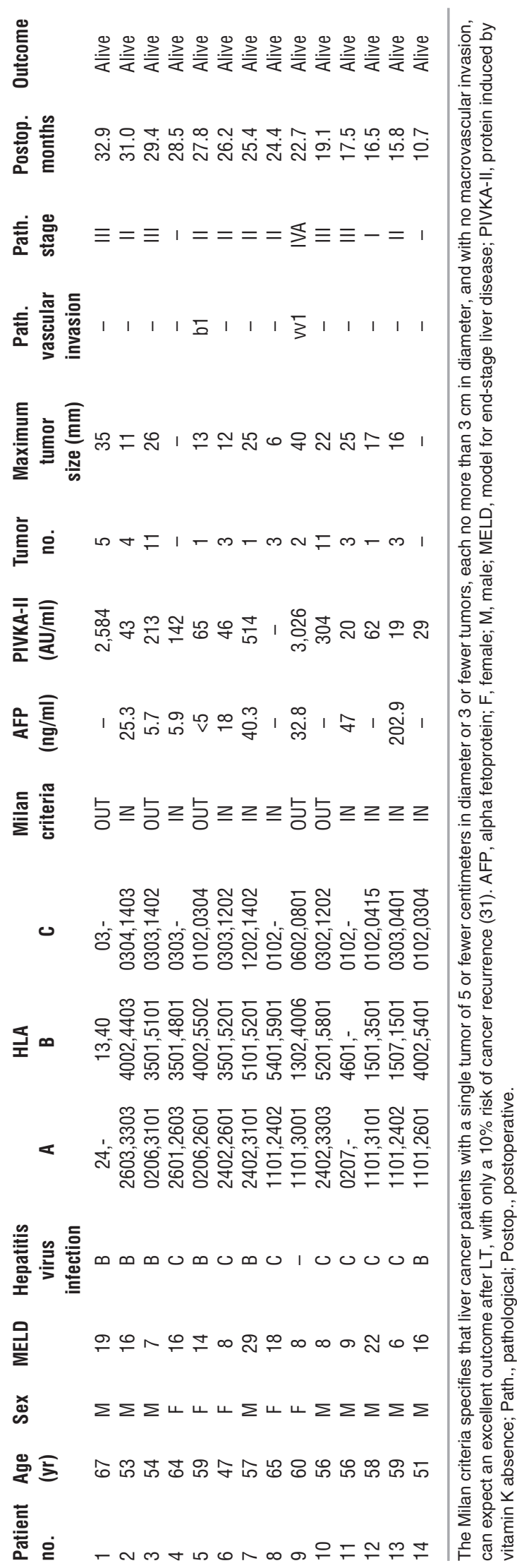

that HCV RNA concentrations sharply decrease a day after LT and increase rapidly thereafter (3). In some of the patients, who did not receive the immunotherapy, HCV RNA titers remained lower than that of the pretransplant titer 1 week after LT, suggesting the individual variation of increasing tempo. However, in almost all patients, HCV RNA titers exceeded the pretransplantation levels by 2 weeks after LT. Notably, HCV infection disappeared in 2 LT recipients after the immunotherapy, but this was not observed in the case of any HCV-infected LT recipients who did not receive the therapy. In one of these patients (who had the lowest HCV RNA levels before LT), HCV RNA has not been detected to date (20 months after LT), even with a qualitative assay. In the other patient, HCV RNA became detectable at 2 months after LT. On the other hand, the 2 patients with the highest $\mathrm{HCV}$ viral loads did not respond at all to the immunotherapy. Thus, the effects of immunotherapy were dependent on the HCV virus load before LT, probably because of the proportion of effectors and targets. All patients with HCV viremia are currently being treated with pegylated IFN- $\alpha 2 b$ and ribavirin. Nevertheless, during the first month after LT, the HCV RNA titers in the sera of LT recipients who received the immunotherapy were statistically lower than those in the sera of $\mathrm{LT}$ recipients who did not receive the therapy $(P<0.05)$ (Figure 3). Among the LT recipients who received the immunotherapy, at 2 weeks after LT, HCV RNA remained undetectable in 4 patients (responders), whereas it was detectable in the other 3 patients (nonresponders). The serum ALT levels did not differ between the responders and nonresponders (Supplemental Figure 1; supplemental material available online with this article; doi:10.1172/JCI38374DS1), suggesting that the immunotherapy did not inhibit HCV RNA by injuring HCV-infected hepatocytes.

In vitro evidence to prove the anti-HCV activity of IL-2/OKT3-treated liver lymphocytes by using HCV replicon-containing hepatic cells. The liver allograft-derived lymphocytes were cultured in complete medium with and without IL-2 for 3 days. This was followed by adding OKT 3 to the culture medium 1 day before coculturing the lymphocytes with HCV replicon-containing hepatic cells in a transwell system, at an indicated time. While the freshly isolated liver allograft-derived lymphocytes inhibited HCV replication in the HCV replicon-containing hepatic cells to some extent, the cultivation of these lymphocytes with IL-2/OKT3 markedly promoted anti-HCV activity. Absence of exposure to either IL-2 or OKT3 resulted in reduced anti-HCV activity of the lymphocytes (OKT3 had a more profound influence than IL-2) (Figure 4A). When the lymphocytes were treated with IL-2 alone, the CD56 ${ }^{+}$fraction, including NK and NKT cells, that had been isolated by magnetic cell sorting inhibited $\mathrm{HCV}$ replication more strongly than the CD56- fraction; further, the $\mathrm{CD}^{-}{ }^{-} \mathrm{CD} 56^{+} \mathrm{NK}$ cell and $\mathrm{CD}^{+}{ }^{+} \mathrm{CD} 56^{+}$ NKT cell subfractions showed equivalent anti-HCV activity (Figure 4, B and C). On the other hand, when the lymphocytes were treated with both IL-2 and OKT3, the CD56 ${ }^{+}$and CD56- fractions showed similar levels of anti-HCV activity (Figure 4B). After the treatment with IL-2 and OKT3, IFN- $\gamma$ was the predominant cytokine in the culture supernatant of the lymphocytes (Figure 5A), and intracellular IFN- $\gamma$ expression was induced in the CD3 ${ }^{-} \mathrm{CD} 56^{+}$ $\mathrm{NK}, \mathrm{CD}^{+} \mathrm{CD}^{-} 6^{+} \mathrm{NKT}$, and $\mathrm{CD} 3^{+} \mathrm{CD}^{-} 6^{-} \mathrm{T}$ cells (Figure $5 \mathrm{~B}$ ). There was no difference between the proportions of TRAIL ${ }^{+}$and TRAIL ${ }^{-}$ $\mathrm{CD}^{-}{ }^{-} \mathrm{CD} 56^{+} \mathrm{NK}$ cells producing IFN- $\gamma$ (Supplemental Figure 2). Adding $\mathrm{mAb}$ against IFN- $\gamma$ to the coculture of lymphocytes with $\mathrm{HCV}$ replicon cells markedly weakened the anti-HCV effects. The incomplete restoration of the anti-HCV effect by anti-IFN- $\gamma$ treat- 
Table 2

Donor and graft characteristics

\begin{tabular}{|c|c|c|c|c|c|c|c|c|c|}
\hline Donor & Donor & Donor & & HLA & & Relationship & Graft & Graft & No. of cells \\
\hline no. & age (yr) & sex & A & B & C & & & weight (g) & administered $\left(\times 10^{6}\right)$ \\
\hline 1 & 41 & M & $24,-$ & 07,40 & 03,07 & Offspring & Right & 608 & 172 \\
\hline 2 & 24 & M & 2402,2603 & 4002,2603 & 0304,5201 & Offspring & Right & 658 & 38 \\
\hline 3 & 51 & $\mathrm{~F}$ & 0201,2402 & 0702,3901 & 0702,- & Spouse & Right & 670 & 129 \\
\hline 4 & 34 & M & 2601,2603 & 4001,4801 & 0303,0401 & Offspring & Left & 414 & 143 \\
\hline 5 & 31 & M & 0206,2402 & 4002,5401 & 0102,0304 & Offspring & Posterior & 702 & 135 \\
\hline 6 & 53 & $\mathrm{~F}$ & $2402,-$ & 5201,5401 & 0102,1202 & Sibling & Right & 538 & 411 \\
\hline 7 & 24 & M & 2601,3101 & 4006,5201 & 0801,1202 & Offspring & Right & 642 & 350 \\
\hline 8 & 34 & M & $1101,-$ & 4001,5401 & 0102,1502 & Offspring & Right & 846 & 229 \\
\hline 9 & 37 & M & 0201,1101 & 1501,4006 & 0702,0801 & Offspring & Left & 402 & 811 \\
\hline 10 & 28 & M & 1101,3303 & 5502,5801 & 0102,0302 & Offspring & Right & 686 & 517 \\
\hline 11 & 28 & M & 0207,2402 & 4601,5201 & 0102,5201 & Offspring & Right & 558 & 414 \\
\hline 12 & 27 & M & 0201,1101 & 1501,3501 & 0303,0415 & Offspring & Right & 628 & 509 \\
\hline 13 & 54 & $\mathrm{~F}$ & 1101,2402 & 1501,1507 & 0303,0401 & Sibling & Right & 650 & 460 \\
\hline 14 & 21 & $F$ & 2601,2603 & 1501,5401 & 0102,0303 & Offspring & Right & 436 & 382 \\
\hline
\end{tabular}

ment suggests the possibility that other inflammatory cytokines may also be responsible for the anti-HCV effect, although we have not defined them at present (Figure $5 \mathrm{C}$ ). Thus, the vigorous antiHCV activity of IL-2/OKT3-treated liver lymphocytes was dependent, at least in part, on their IFN- $\gamma$-secreting activity.

IFN- $\gamma$-secreting activity in LT recipients after adoptive immunotherapy. At 14 days after LT, the number of IFN- $\gamma$-secreting cells in the peripheral blood of LT recipients who received adoptive immunotherapy was significantly higher than that in the peripheral blood of $L T$ recipients who did not receive immunotherapy during the trial period (Figure 6). This result was consistent with the results of the in vitro studies showing the crucial role of IFN- $\gamma$ produced in IL-2/OKT3-treated liver lymphocytes.

In vivo evidence to prove the anti-HCV activity of adoptive immunotherapy by using HCV-infected human hepatocyte-chimeric mice. HCV-infected mice have previously been developed by inoculating $\mathrm{HCV}$-infected human serum into chimeric urokinase-type plasminogen activatorSCID (uPA-SCID) mice with engrafted human hepatocytes (18). This HCV-infected mouse model has been reported to be useful for evaluating anti-HCV drugs such as IFN- $\alpha$ and anti-NS3 protease (19). We also generated a human hepatocyte-chimeric mouse model, in which mouse hepatocytes were almost completely replaced by human hepatocytes (20). These mice consistently developed long-term HCV infections, showing high viral titers after inoculation with HCV genotype $1 \mathrm{~b}$-infected human serum $(50 \mu \mathrm{l} /$ mouse) (Supplemental Figure 3). Intraperitoneal injection of IL-2/ OKT3-treated liver lymphocytes $\left(20 \times 10^{6}\right.$ cells/mouse $)$, at 2 weeks after inoculation with the infected serum, consistently prevented the development of HCV infection in the human hepatocyte-chimeric mice (Figure 7A). Such anti-HCV effects were countered by anti-IFN- $\gamma$ neutralizing antibodies in some chimeric mice, suggesting the potential role played by IFN- $\gamma$ in the anti-HCV effects of the immunotherapy. The administration of recombinant human IFN- $\gamma$ markedly and consistently prevented the development of HCV infection in the human hepatocyte-chimeric mice. Once the HCV RNA became undetectable in the sera of chimeric mice receiving either IL-2/OKT3-treated liver lymphocytes or recombinant IFN- $\gamma$, it could not be detected again. The constant levels of human serum albumin in the chimeric mice indicated that neither the immunotherapy nor recombinant IFN- $\gamma$ administration had significant adverse effects on human hepatocytes in those mice (Figure 7B). Once HCV infection had developed in the human hepatocyte-chimeric mice, who showed high titers of $\mathrm{HCV}$ RNA in their sera (over $10^{3}$ copies $/ \mathrm{ml}$ ) 4 weeks after the inoculation of $\mathrm{HCV}$-infected serum, the preventive effects of the adoptive immunotherapy or recombinant IFN- $\gamma$ on HCV infection were no longer observed (Figure 7C).
Table 3

Characteristics of HCV-infected LT recipients that received and did not receive immunotherapy

\begin{tabular}{|c|c|c|c|c|c|c|c|}
\hline No. & Age & Sex & $\begin{array}{c}\text { HCV } \\
\text { genotype }\end{array}$ & MELD & $\begin{array}{c}\text { Pre-HCV RNA } \\
(\mathrm{KIU} / \mathrm{ml})\end{array}$ & $\begin{array}{l}\text { Postoperative } \\
\text { months }\end{array}$ & Immunosuppressant \\
\hline \multicolumn{8}{|c|}{ With immunotherapy } \\
\hline 4 & 64 & $\mathrm{~F}$ & $1 b$ & 16 & 210 & 29 & Basiliximab+FK506+MMF \\
\hline 6 & 47 & $\mathrm{~F}$ & $1 b$ & 8 & 5,000 & 26 & Basiliximab+CsA+MMF \\
\hline 8 & 65 & $\mathrm{~F}$ & $1 b$ & 18 & 2,400 & 24 & Basiliximab+CsA+MMF \\
\hline 10 & 56 & M & $1 b$ & 8 & 970 & 19 & Basiliximab+FK506+MMF \\
\hline 11 & 56 & $M$ & $1 b$ & 9 & 1,700 & 17 & Basiliximab+FK506+MMF \\
\hline 12 & 58 & M & $1 b$ & 22 & 19 & 17 & Basiliximab+FK506+MMF \\
\hline 13 & 59 & $\mathrm{M}$ & $1 b$ & 6 & 2,200 & 16 & Basiliximab+FK506+MMF \\
\hline \multicolumn{8}{|c|}{ Without immunotherapy } \\
\hline A & 51 & $M$ & $1 b$ & 27 & 420 & 42 & Basiliximab+FK506+MMF \\
\hline B & 44 & $M$ & $1 b$ & 10 & 1,600 & 32 & Basiliximab+FK506+MMF \\
\hline C & 54 & M & $1 b$ & 8 & 180 & 22 & Basiliximab+CsA+MMF \\
\hline D & 56 & $M$ & $2 \mathrm{a}$ & 10 & 470 & 20 & Basiliximab+FK506+MMF \\
\hline E & 57 & $M$ & $1 b$ & 12 & 3,200 & 6 & Basiliximab+FK506+MMF \\
\hline
\end{tabular}



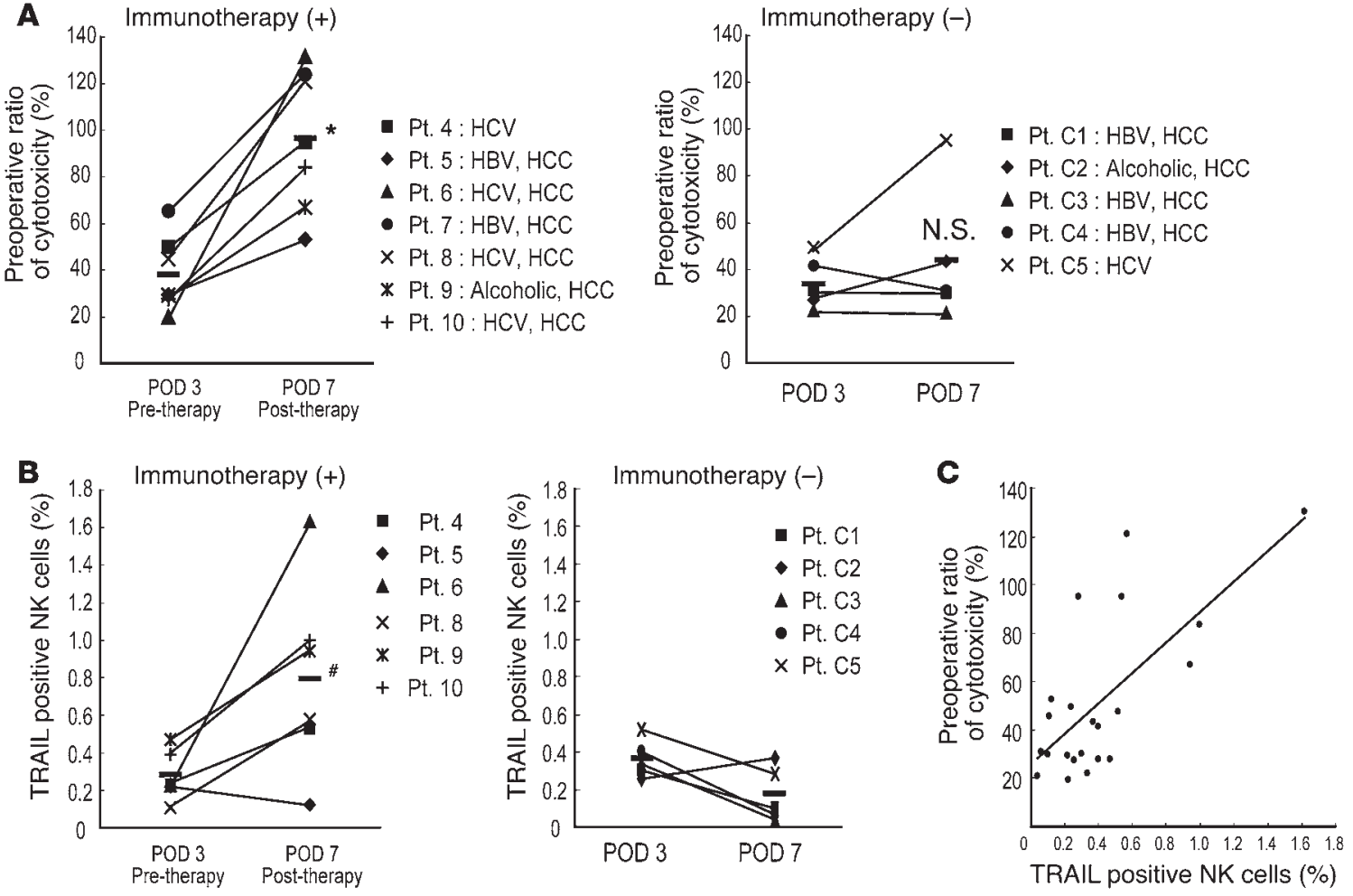

Figure 2

Adoptive immunotherapy with IL-2/OKT3-treated liver lymphocytes promoted the cytotoxic activity and TRAIL expression of NK cells in LT recipients. (A) The NK cytotoxic activities of the indicated effectors against their target cells were analyzed by the ${ }^{51} \mathrm{Cr}$-release assay. The dot plot represents the NK cytotoxic activities of freshly isolated peripheral blood lymphocytes obtained from recipients who received immunotherapy (+) $(n=7)$ and did not receive immunotherapy $(-)(n=5)$ against HepG2 target cells (effector/target [E/T] ratio, $40: 1) 3$ and 7 days after LT. NK cytotoxic activities are represented as a proportion (percentage) of the preoperative cytotoxicity in each patient. Horizontal lines indicate the mean. Statistical analyses were performed using the 2 -tailed, paired Student's $t$ test. ${ }^{*} P<0.05$ for day 7 versus day 3 . (B) The frequency of TRAIL ${ }^{\text {NKK }}$ cells increased remarkably in the peripheral blood of LT recipients who received the immunotherapy. Horizontal lines indicate the mean. Statistical analyses were performed using the Mann-Whitney $U$ test. ${ }^{\#} P=0.013$ for immunotherapy group versus untreated group in postoperative day 7 . (C) Correlation between TRAIL+ NK cell ratio and NK cytolytic activity after LT (Spearman rank-order correlation coefficient $=0.54, P=0.01$ ). Statistical analyses were performed using the Spearman rank-order correlation coefficient. The diagonal line indicates a linear regression line. Each dot indicates the cytotoxicity and TRAIL+ NK cell percentange of each patient. C1, control 1; POD, postoperative day; Pt., patient.

\section{Discussion}

The consequences of recurrent hepatitis $\mathrm{C}$ on the survival of graft and $\mathrm{LT}$ recipients can only be avoided by the development of safe and effective antiviral strategies that can not only prevent initial graft infection but also eradicate established hepatitis $C$ recurrence $(3,4)$. With regard to initial graft infection, the circulating virions infect the liver graft immediately after LT. HCV RNA concentrations usually increase a few days after LT, reflecting active $\mathrm{HCV}$ replication in the liver graft. In general, in such an early phase of a viral infection, the first line of host defense may be effective in removing the virus; however, recent reports have indicated that $\mathrm{HCV}$ effectively escapes the innate immune system comprising NK and NKT cells, resulting in persistent infection $(21,22)$. It has been reported that cross-linking of CD81 on NK cells by the major envelope protein of HCV, HCV-E2, blocks NK cell activation, IFN- $\gamma$ production, cytotoxic granule release, and proliferation (21). Engagement of CD81 on NK cells blocks tyrosine phosphorylation through a mechanism that is distinct from the negative signaling pathways associated with NK cell inhibitory receptors for major histocompatibility complex class I molecules (22). These facts prove that HCV-E2-mediated inhibition of NK cells is an efficient $\mathrm{HCV}$ evasion strategy, which involves targeting the early antiviral activities of NK cells and allowing the virus to establish itself as a chronic infection.

We have explored whether CD81 cross-linking-induced inhibitory effects occur even in IL-2-stimulated NK cells. CD81 cross-linking by a mAb specific for CD81 inhibited antitumor cytotoxicity and anti-HCV activity mediated by resting NK cells, but this manipulation did not alter both these activities of IL-2-stimulated NK cells (Supplemental Figure 4). This indicated that exposure to IL-2 before CD81 cross-linking abrogates subsequent inhibitory signals in the NK cells. This would be one mechanism whereby the adoptive immunotherapy with IL-2/OKT3-treated liver lymphocytes inhibited HCV replication at the early phase of infection after LT.

Although the role of NK cells in controlling HCV infection and replication has not been completely elucidated, a recent report has indicated that NK cells do not exert a direct cytolytic effect on the $\mathrm{HCV}$ replicon-containing hepatic cells but release IFN- $\gamma$, suppressing HCV RNA expression (11). The role of IFN- $\gamma$ in the expression of NK cell-mediated anti-HCV activity has been proved by the observa- 

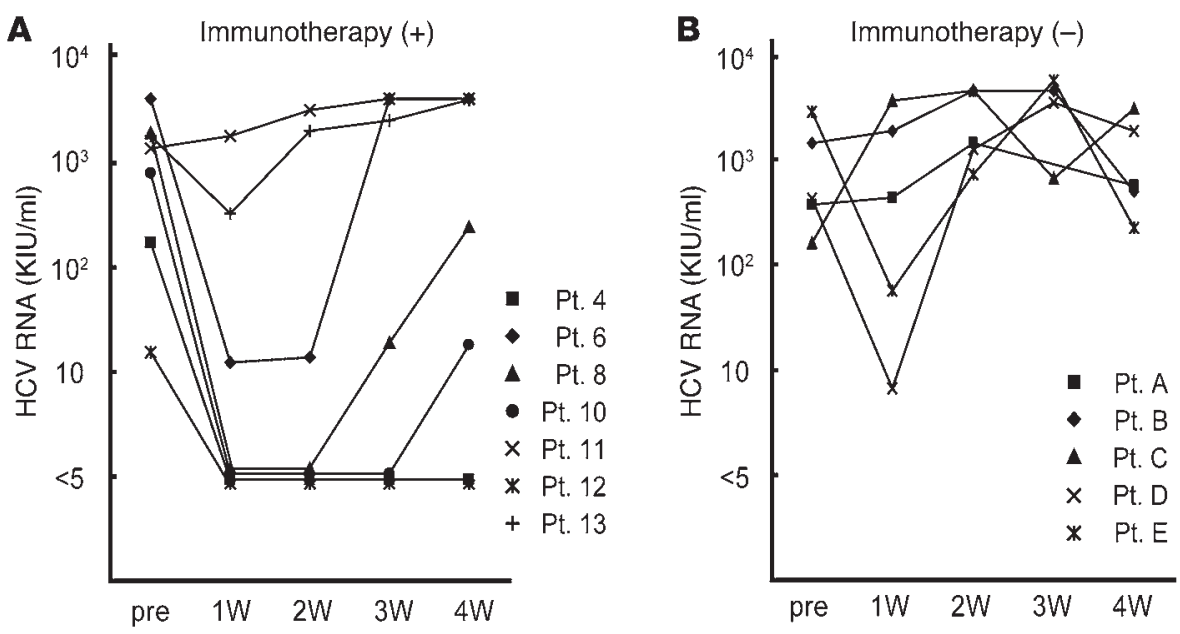

Figure 3

Serial measurement of the HCV RNA titers of $L T$ recipients after $L T$. The HCV RNA titers in the sera of $L T$ recipients who received immunotherapy were markedly lower than those in the sera of LT recipients who did not receive the therapy during the first month after LT. Each line with a different symbol represents serial HCV RNA titers from an $L T$ recipient who received $(+)$ $(\mathbf{A} ; n=7)$ and 1 who did not receive $(-)(\mathbf{B}$; $n=5)$ the immunotherapy after LT. KIU, kilo international unit; pre, pre LT; W, week. tion that NK cell-conditioned media have an enhanced expression of signal transducer and activator of transcription 1, a nuclear factor that is essential in IFN- $\gamma$-mediated antiviral pathways. It has also been reported that hepatocytes cultured in NK cell-conditioned media express higher levels of IFN- $\alpha / \beta$, IFN regulatory factor 3 , and IFN regulatory factor 7 , confirming that NK cells play a key role in suppressing HCV infection of and replication in human hepatocytes in an IFN-dependent manner (23). Similar to recent reports, in the present study, we demonstrated that the NK cells among the IL-2/ OKT3-treated liver lymphocytes released soluble factors, predominantly IFN- $\gamma$, thus suppressing HCV replication (Figures 5-7).

In addition to NK cells, NKT cells are thought to be involved in eliciting innate responses against infection; however, the role of NKT cells in controlling HCV infection/replication remains unclear. One report has indicated that the number of NKT cells in patients with chronic HCV infection does not differ from that in healthy donors; however, activated NKT cells in HCVinfected patients produce higher levels of IL-13 - but comparable levels of IFN- $\gamma$ - than those in healthy subjects, showing that NKT cells are biased toward T-helper 2-type responses in chronic HCV infection (24). Another recent report has shown that the sustained response of patients with chronic hepatitis $\mathrm{C}$ to treatment with IFN- $\alpha$ and ribavirin is closely associated with increased dynamism of NK and NKT cells in the liver, implicating an NKT cell-mediated mechanism in anti-HCV activity (25). Here, we have described that NKT as well as NK cells in the IL-2/
A

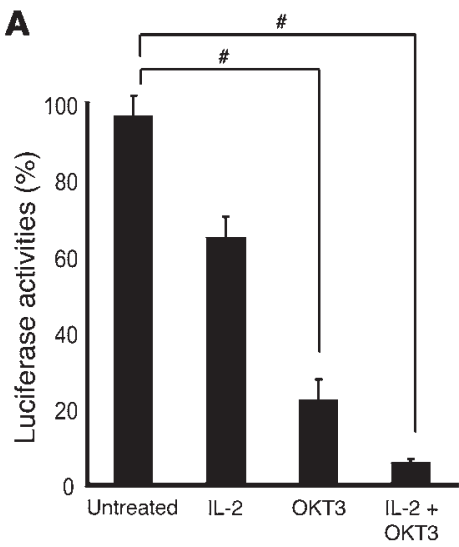

B

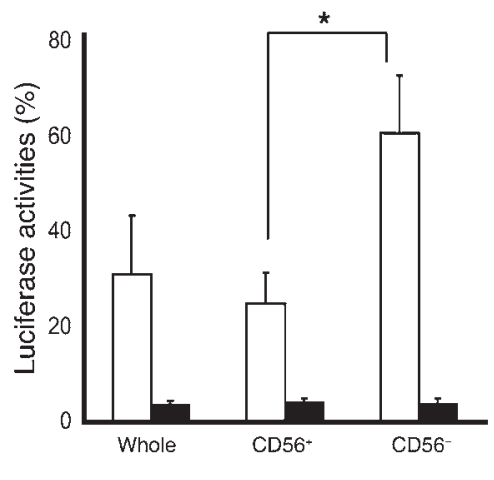

C

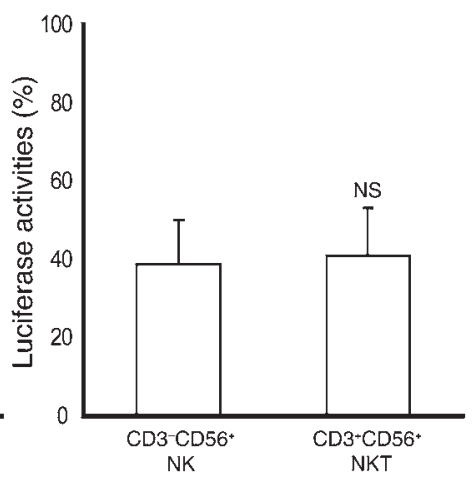

\section{Figure 4}

The cultivation of liver lymphocytes with IL-2/OKT3 markedly promoted anti-HCV activity. (A) Activation by IL-2 and OKT3 significantly promoted the anti-HCV effect of the liver allograft-derived lymphocytes that were cultured in complete medium with and without IL-2 (100 $\mathrm{JRU} / \mathrm{ml})$ for 3 days. OKT3 $(1 \mu \mathrm{g} / \mathrm{ml})$ was then added 1 day before coculturing with HCV replicon cells, at the indicated time. The bar graphs indicate the luciferase activities of the cells in each group. Data are presented as mean \pm SEM $(n=5)$. Statistical analyses were performed using the Mann-Whitney $U$ test with Bonferroni correction after the Kruskal-Wallis $H$ test. $\#<0.01$ for OKT3 and IL-2/OKT3 treatment versus no treatment. (B) CD56+ fraction, including NK and NKT cells, strongly inhibited HCV replication. The culture conditions are described in $\mathbf{A}$. By magnetic cell sorting, $\mathrm{CD}_{5} 6^{+}$and CD56- fractions were isolated from the activated lymphocytes and analyzed for anti-HCV activity. The bar graphs indicate the luciferase activities of the cells in each group (IL-2-treated group, white bars; IL-2 plus OKT3-treated group, black bars). Whole, whole lymphocytes. Data are presented as mean \pm SEM $(n=5)$. Statistical analyses were performed using the Mann-Whitney $U$ test. ${ }^{*} P<0.05$ for $\mathrm{CD} 6^{+}$fraction versus CD56- fraction. (C) Anti-HCV effect of NK cells was almost identical to that of NKT cells after IL-2 activation. The liver allograft-derived lymphocytes were cultured in complete medium with IL-2 (100 JRU/ml) for 3 days. By magnetic sorting, $\mathrm{CD}^{-} \mathrm{CD}_{56}{ }^{+}(\mathrm{NK})$ and $\mathrm{CD}^{+}{ }^{+} \mathrm{CD} 56^{+}(\mathrm{NKT})$ fractions were isolated from the activated lymphocytes and analyzed for anti-HCV activity. Data are presented as mean $\pm \operatorname{SEM}(n=6)$. 
A

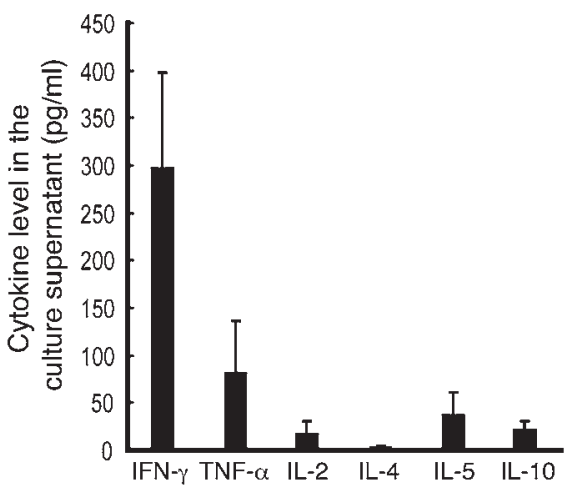

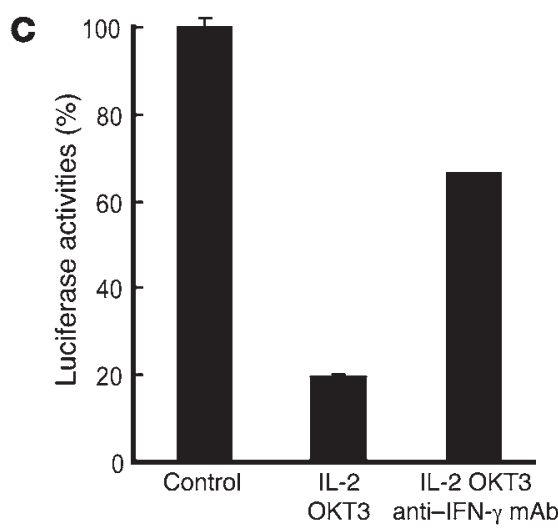

B
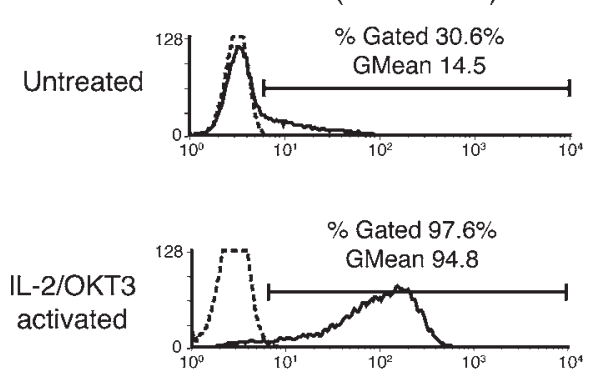

NKT $\left(\right.$ CD3 $\left.{ }^{+} \mathrm{CD} 56^{+}\right)$
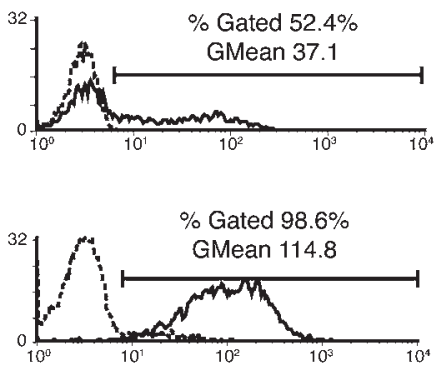

Human IFN- $\gamma$
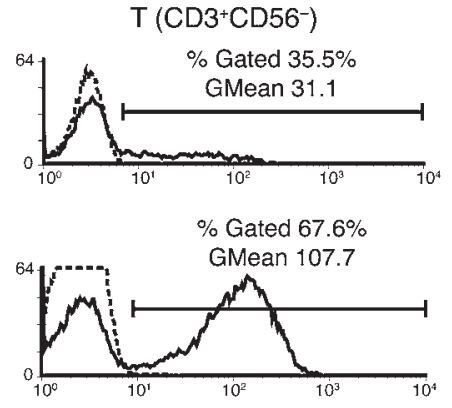

Figure 5

Anti-HCV activity of IL-2/OKT3-treated liver lymphocytes was dependent on their IFN- $\gamma$ secretion ability. (A) IFN- $\gamma$ was the major cytokine released from the cultured cells. The bar graphs indicate the concentrations of various cytokines (IFN- $\gamma$, TNF- $\alpha$, IL-2, IL-4, IL-5, and IL-10) detected in the coculture supernatant by CBA. Data are presented as mean \pm SEM $(n=3)$. (B) The effects of IL-2 and OKT3 (100 JRU/ml and $1 \mu \mathrm{g} / \mathrm{ml}$, respectively) on IFN- $\gamma$ production by stimulated CD3-CD56 ${ }^{+} \mathrm{NK}, \mathrm{CD}^{+} \mathrm{CD} 56^{+} \mathrm{NKT}$, and CD3 ${ }^{+} \mathrm{CD} 56^{-} \mathrm{T}^{-}$cells were evaluated by a combination of cell surface and cytoplasmic mAb staining and subsequent flow cytometric analysis. Histograms represent the log fluorescence intensities obtained upon staining for IFN- $\gamma$ after gating of each fraction. Dotted lines represent negative control staining with isotype-matched mAbs. Horizontal lines indicate the gated portion of lymphocytes. GMean, geometric mean fluorescent intensity. (C) Blocking of IFN- $\gamma$ with mAb $(100 \mu \mathrm{g} / \mathrm{ml})$ elucidated the marked role played by IFN- $\gamma$ in producing the anti-HCV effect. The bar graphs indicate the luciferase activities of the cells in each group. Data are presented as mean \pm SEM of a representative triplicate sample.

OKT3-treated liver lymphocytes could play a vital role in controlling HCV replication in hepatic cells via an IFN- $\boldsymbol{\gamma}$-associated mechanism (Figures 5 and 6).

Therefore, in the early phase of HCV reinfection after LT, the effects of IFN- $\gamma$ secretion from adoptively injected liver lymphocytes may include inhibition of HCV virion production, which is probably caused by suppression of viral RNA and protein synthesis without immune lysis of intact hepatic cells. This IFN- $\gamma$ secretion from both $\mathrm{CD}^{+} \mathrm{CD}^{+} 6^{+} \mathrm{NKT}$ cells and $\mathrm{CD}^{+} \mathrm{T}$ cells was markedly upregulated after treatment with OKT3, which was originally used to prevent GVHD (Figure 5B). This is possibly because of the potent mitogenic activity of OKT3 that induces the activation of $\mathrm{CD}^{+}{ }^{+} \mathrm{CD} 56^{+} \mathrm{NKT}$ cells and $\mathrm{CD}^{+} \mathrm{T}$ cells. However, the administration of OKT3-coated cells in vivo results in the opsonization and subsequent trapping and/or lympholysis of cells by the reticuloendothelial system (26-28). Thus, GVHD is prevented in LT recipients treated with adoptive immunotherapy.

Our finding that the IL-2/OKT3-treated liver lymphocytes controlled HCV replication via an IFN- $\gamma$-associated mechanism can lead to the clinical application of recombinant IFN- $\gamma$ for anti-HCV treatment. However, a clinically applicable dose of recombinant IFN- $\gamma$ could not induce significant inhibitory effects on $\mathrm{HCV}$ viremia in the previous study (29). Based on the accumulation of adoptively injected IL-2/OKT3-treated liver lymphocytes in the liver of human hepatocyte-chimeric mice (data not shown), the immunotherapy with the liver lymphocytes would provide sufficient IFN- $\gamma$ to the HCV-infected site.

It has been recently reported that $\mathrm{HCV}$-specific $\mathrm{CD}^{+} \mathrm{T}$ cells exert strong antiviral effects by both cytopathic and IFN- $\gamma$-mediated noncytopathic effector functions (30). However, in patients with chronic HCV infection, dysfunction and functional restoration of $\mathrm{HCV}$-specific $\mathrm{CD}^{+} \mathrm{T}$ cell responses have been reported (31). Since $\mathrm{HCV}$-specific $\mathrm{CD}^{+} \mathrm{T}$ cell defects may be important in persistent $\mathrm{HCV}$ infections, correcting these defects is considered to our knowledge to be a novel approach to treat HCV infection. Further studies are required to investigate whether activation of NK or NKT cells functionally restores $\mathrm{HCV}$-specific $\mathrm{CD} 8^{+} \mathrm{T}$ cells.

In conclusion, adoptive immunotherapy using IL-2/OKT3treated liver lymphocytes containing abundant NK and NKT cells could mount remarkable anti-HCV responses in HCV-infected LT recipients, although its effects were incomplete or transient. Treatment-related improvements, such as defining the best schedule and frequency of cell inoculation and developing more potent effectors, could improve clinical benefits. 


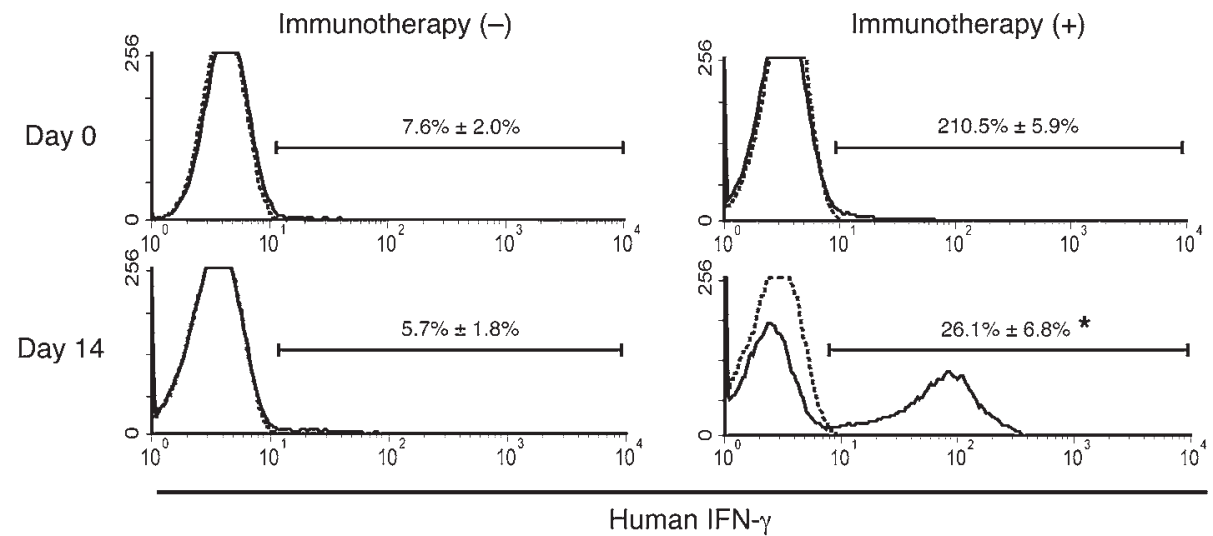

Figure 6

Adoptive immunotherapy with IL-2/OKT3-treated liver lymphocytes induced the production of IFN- $\gamma$ in the LT recipients. At 14 days after LT, the number of IFN- $\gamma$-secreting cells in the peripheral blood of LT recipients treated with the adoptive immunotherapy (+) with IL-2/OKT3-treated liver lymphocytes, including NK and NKT cells, was significantly higher than that in the peripheral blood of untreated LT recipients (-). Histograms represent the proportion (percentage) of IFN- $\gamma$-positive cells among the mononuclear cells obtained from the peripheral blood of the immunotherapy $(n=4)$ and control group $(n=4)$ LT recipients. Dotted lines represent negative control staining with isotype-matched mAbs. Horizontal lines indicate the gated portion of lymphocytes. Data are presented as mean \pm SEM. Histogram profiles shown are representative of 4 independent experiments. Statistical analyses were performed using the Mann-Whitney $U$ test. ${ }^{\star} P<0.05$ for immunotherapy group versus control group.

\section{Methods}

Subjects. All the human liver samples were collected at Hiroshima University Hospital. Tissue specimens were collected after approval from the Institutional Review Board of Hiroshima University and after written informed consent was obtained from the patients. The use of immunotherapy with IL-2/ OKT3-treated liver lymphocytes was approved by the Clinical Institutional Ethical Review Board of Hiroshima University. Written informed consent was obtained from all of the patients. This approach was successfully used in 14 cirrhotic patients with HCC undergoing clinical LT (Tables 1 and 2). Of these 14 patients, 7 had chronic $\mathrm{HCV}$ infection. Five other $\mathrm{LT}$ recipients with chronic $\mathrm{HCV}$ infection did not agree to receive this immunotherapy during the trial period. HCV RNA was qualitatively detected in the sera of these patients by a standardized qualitative RT-PCR assay (Amplicor HCV monitor, version 2.0; Roche Diagnostics) every week during the first month after LT.
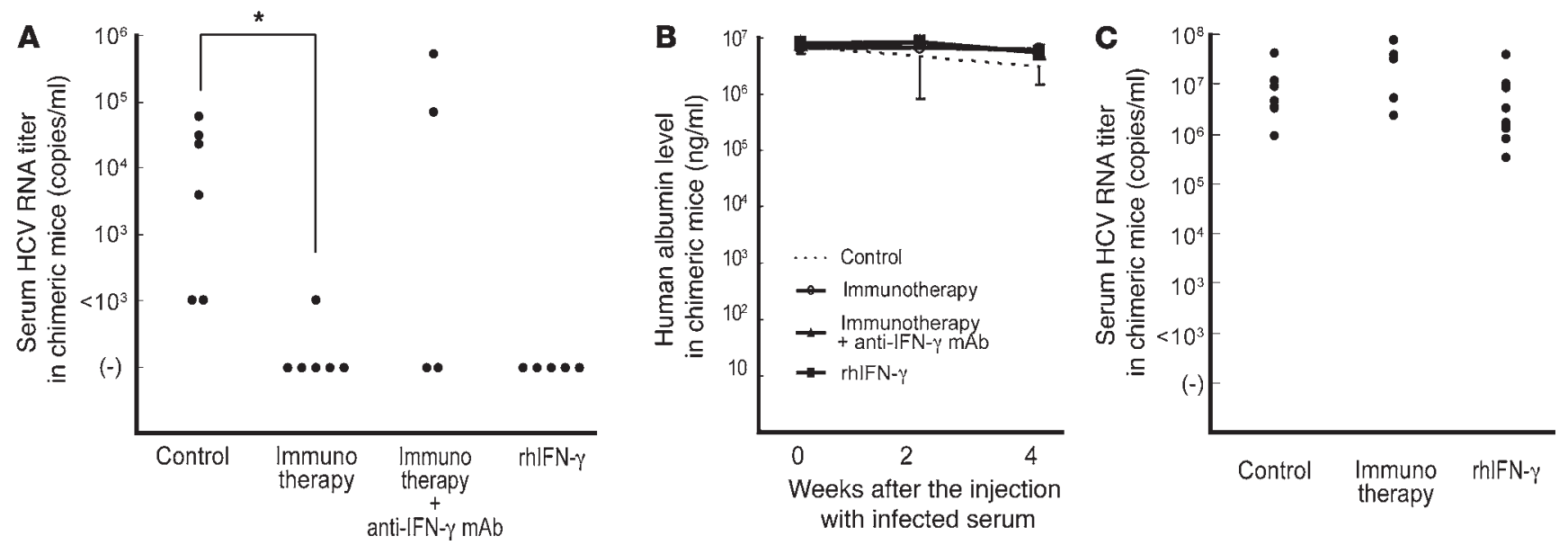

Figure 7

Adoptive immunotherapy with IL-2/OKT3-treated liver lymphocytes prevented HCV infection in human hepatocyte-chimeric mice. (A) Human hepatocyte-chimeric mice were intravenously injected with human serum samples positive for HCV genotype $1 \mathrm{~b}$. Two weeks after injecting the infected serum, the mice were intraperitoneally inoculated with IL-2/OKT3-treated liver lymphocytes $\left(20 \times 10^{6}\right.$ cells/mouse; $\left.n=6\right)$ for adoptive immunotherapy. When indicated, anti-human IFN- $\gamma$ mAb was injected intraperitoneally 1 day before the immunotherapy $(n=4)$. Intraperitoneal injection of recombinant human IFN- $\gamma($ rhIFN- $\gamma$ ) was commenced at 2 weeks after injecting the infected serum $(n=5)$. The untreated mice served as controls $(n=6)$. The dot plots represent serum HCV RNA titers in each chimeric mouse 4 weeks after the injecting the infected serum. Statistical analyses were performed using the Mann-Whitney $U$ test. ${ }^{\star} P<0.01$ for immunotherapy group versus control group. (B) The lines represent serial changes in human serum albumin levels in the sera of the mice indicated above. Data are presented as mean \pm SEM. (C) IL-2/OKT3-treated liver lymphocytes $\left(20 \times 10^{6}\right.$ cells/mouse) were intraperitoneally inoculated 4 weeks after the injection with the infected serum $(n=5)$ for adoptive immunotherapy. Intraperitoneal injection of recombinant human IFN- $\gamma$ was commenced 4 weeks after the injecting the infected serum $(n=9)$. The untreated mice served as controls $(n=9)$. The dot plots represent serum HCV RNA titers in each chimeric mouse 6 weeks after injection with the infected serum. 
Isolation of lymphocytes from liver allograft perfusate. Donor hepatectomy and the transplantation procedure were performed as described previously (32). After hepatectomy, ex vivo perfusion of the liver allograft was performed through the portal vein. Liver allograft-derived lymphocytes were isolated by gradient centrifugation with Ficoll-Paque (GE Healthcare Bio-Sciences AB).

Adoptive transfer of IL-2/OKT3-treated liver lymphocytes. Liver lymphocytes were cultured with human recombinant IL-2 (100 Japanese reference units $/ \mathrm{ml}$ [JRU/ml]; Takeda) in complete medium at $37^{\circ} \mathrm{C}$ in a $5 \% \mathrm{CO}_{2}$ incubator for 3 days. One day before the infusion, $1 \mu \mathrm{g} / \mathrm{ml}$ of OKT3 (Janssen-Kyowa) was added in order to opsonize the $\mathrm{CD}^{+}$fraction. On the day of infusion, the cells were washed twice with $0.9 \%$ sodium chloride and resuspended with $5 \%$ human serum albumin in $0.9 \%$ sodium chloride for injection (Figure 1). The viability of the cells was assessed by the dye-exclusion test, and the cells were checked twice for possible contamination by bacteria, fungi, and endotoxins.

Cytotoxicity assay. ${ }^{51} \mathrm{Cr}$-release assay was done as previously described (5), using HepG2 tumor cells (Japanese Cancer Research Resources Bank) as targets. Briefly, ${ }^{51} \mathrm{Cr}$-labeled target tumor cells were added for 4 hours at $37^{\circ} \mathrm{C}$ to effector cells in round-bottomed 96 -well microtiter plates (BD Biosciences - Discovery Labware). The percentage of specific ${ }^{51} \mathrm{Cr}$ release was calculated as follows: \% cytotoxicity $=[(\mathrm{cpm}$ of experimental release - cpm of spontaneous release)/(cpm of maximum release - cpm of spontaneous release) $] \times 100$. All the assays were performed in triplicate.

Flow cytometry. Flow cytometric analyses were performed using a FACSCalibur dual-laser cytometer (BD Biosciences). The following mAbs were used for the surface staining of the lymphocytes: FITC-conjugated antiCD3 mAb (clone HIT3a; BD Biosciences - Pharmingen); PE-conjugated anti-CD56 mAb (clone B159; BD Biosciences - Pharmingen); and biotinylated anti-TRAIL (biotin-conjugated anti-TRAIL) mAb (clone RIK-2; eBioscience). The biotinylated $\mathrm{mAb}$ was visualized using APC-streptavidin (BD Biosciences - Pharmingen). Dead cells identified by light scatter and propidium iodide staining were excluded from the analysis. IFN- $\gamma$ production in the lymphocytes was measured by a combination of cell surface and cytoplasmic $\mathrm{mAb}$ staining and subsequent flow cytometric analysis, as described previously (33).

Isolation of $\mathrm{CD}^{-} 6^{+}$and $\mathrm{CD56} 6^{-}$fractions and that of $\mathrm{NK}$ and NKT cells. Liver allograft-derived lymphocytes were separated into a $\mathrm{CD} 56^{+}$fraction including NK and NKT cells - and a CD56- fraction by using auto MACS (Miltenyi Biotec) with anti-human CD56 microbeads (Miltenyi Biotec) according to the manufacturer's instructions. The NK and NKT cells were also isolated by magnetic cell sorting, using the human NK cell isolation kit or human $\mathrm{CD}^{+} \mathrm{CD}^{+} 6^{+} \mathrm{NKT}$ cell isolation kit (Miltenyi Biotec). The purity of the isolated fractions was assessed by flow cytometric analysis, and only the fractions with purities greater than $90 \%$ were used for functional studies.

Coculture with HCV replicon-containing hepatic cells. An HCV subgenomic replicon plasmid, pRep-Feo, was derived from pRep-Neo (originally, pHCVIbneo-delS; ref. 34). The pRep-Feo carries a fusion gene comprising firefly luciferase (Fluc) and neomycin phosphotransferase, as described elsewhere $(35,36)$. After culture in the presence of G418 (Invitrogen), pRep-Feo cell lines stably expressing the replicons were established. For coculture experiments, transwell tissue culture plates (pore size, $1 \mu \mathrm{m}$; Costar) were used. $\mathrm{HCV}$ replicon-containing hepatic cells $\left(10^{5}\right.$ cells) were incubated in the lower compartment with different numbers of lymphocytes in the upper compartment. The hepatic cells in the lower compartments were collected 48 hours after coculture for the luciferase assay. Luciferase activities were measured with a luminometer (Lumat LB9501; Promega), using the BrightGlo Luciferase Assay System (Promega).

Cytometric bead array. Cytokine (IFN- $\gamma$, TNF- $\alpha$, IL-2, IL-4, IL-5, IL-10) levels in the coculture assay supernatants were measured with the FACSCalibur duallaser cytometer (BD Biosciences), using a BD Human Th1/Th2 Cytokine Cytometric Bead Array (CBA) Kit according to the manufacturer's instructions.

Generation of human hepatocyte-chimeric mice. Generation of the $u \mathrm{PA}^{+/+}$ $S C I D^{+/+}$mice and transplantation of human hepatocytes were performed as described recently by our group $(20,37)$. Mouse serum concentrations of human serum albumin correlated with the repopulation index (20), and these were measured as described previously (37).

In vivo studies using human hepatocyte-chimeric mice. Human hepatocyte-chimeric mice were intravenously injected with $50 \mu$ l of the human serum samples positive for HCV genotype $1 \mathrm{~b}$. The serum HCV RNA titer in human hepatocyte-chimeric mice was detected by nested PCR, as previously described $(38,39)$. All animal protocols described in this study were performed in accordance with the guidelines and with approval of the Ethics Review Committee of Animal Experimentation of the Graduate School of Biomedical Sciences, Hiroshima University. Either 2 or 4 weeks after injecting the infected serum, the mice were intraperitoneally inoculated with IL-2/ OKT3-treated liver lymphocytes $\left(20 \times 10^{6}\right.$ cells/mouse) for adoptive immunotherapy. When indicated, anti-human IFN- $\gamma$ mAb (R\&D Systems) (1.5 $\mathrm{mg} /$ mouse) was injected intraperitoneally 1 day before the immunotherapy. In a separate experiment, intraperitoneal injection of recombinant human IFN- $\gamma$ (Imunomax- $\gamma$; Shionogi \& Co. Ltd.) was commenced at either 2 or 4 weeks after injecting the infected serum. IFN- $\gamma$ was administered as follows: $1 \times 10^{5} \mathrm{IU}$ on the first day and thereafter $2 \times 10^{4} \mathrm{IU} /$ day for 13 days.

Statistics. Data are presented as mean \pm SEM. The statistical differences of the results were analyzed by 2-tailed, paired Student's $t$ test, Mann-Whitney $U$ test, and Mann-Whitney $U$ test with Bonferroni correction after the Kruskal-Wallis $H$ test, using the Stat View program. $P$ values of 0.05 or less were considered statistically significant.

\section{Acknowledgments}

This work was supported in part by a Grant-in-Aid for Exploratory Research (19659323) from the Japan Society for the Promotion of Science. We thank Kentaro Ide, Toshimitsu Irei, Hiroyuki Tahara, Masataka Banshodani, Nabin Basnet, Hirofumi Tazawa, and Keiko Kajitani for their advice and encouragement and Yuko Ishida for expert technical assistance.

Received for publication December 17, 2008, and accepted in revised form July 29, 2009.

Address correspondence to: Hideki Ohdan, Department of Surgery, Division of Frontier Medical Science, Programs for Biomedical Research, Graduate School of Biomedical Sciences, Hiroshima University, 1-2-3 Kasumi, Minami-ku, Hiroshima 734-8551, Japan. Phone: 81-82-257-5220; Fax: 81-82-257-5224; E-mail: hohdan@ hiroshima-u.ac.jp. Or to: Kazuaki Chayama, Department of Medicine and Molecular Science, Division of Frontier Medical Science, Programs for Biomedical Research, Graduate School of Biomedical Sciences, Hiroshima University, 1-2-3 Kasumi, Minami-ku, Hiroshima 734-8551, Japan. Phone: 81-82-257-5190; Fax: 81-81257-5194; E-mail: chayama@hiroshima-u.ac.jp.

\footnotetext{
1. Petrovic, L.M. 2006. Early recurrence of hepatitis C virus infection after liver transplantation. Liver Transpl. 12:S32-S37.

2. Brown, R.S. 2005. Hepatitis C and liver transplantation. Nature. 436:973-978.
}

3. Garcia-Retortillo, M., et al. 2002. Hepatitis C virus kinetics during and immediately after liver transplantation. Hepatology. 35:680-687.

4. Berenguer, M. 2002. Natural history of recurrent hepatitis C. Liver Transpl. 8:S14-S18.
5. Ishiyama, K., et al. 2006. Difference in cytotoxicity against hepatocellular carcinoma between liver and periphery natural killer cells in humans. Hepatology. 43:362-372.

6. Ohira, M., et al. 2006. Adoptive transfer of TRAIL- 
expressing natural killer cells prevents recurrence of hepatocellular carcinoma after partial hepatectomy. Transplantation. 82:1712-1719.

7. Miller, J.S., et al. 2005. Successful adoptive transfer and in vivo expansion of human haploidentical NK cells in patients with cancer. Blood. 105:3051-3057.

8. Hirata, M., et al. 1998. Increase in natural killer cell activity following living-related liver transplantation. Transpl. Int. 11(Suppl. 1):S185-S188.

9. Harada, N., et al. 2004. IL-12 gene therapy is an effective therapeutic strategy for hepatocellular carcinoma in immunosuppressed mice. J. Immunol. 173:6635-6644.

10. Golden-Mason, L., and Rosen, H.R. 2006. Natural killer cells: primary target for hepatitis $\mathrm{C}$ virus immune evasion strategies? Liver Transpl. 12:363-372.

11. Li, Y., et al. 2004. Natural killer cells inhibit hepatitis C virus expression. J. Leukoc. Biol. 76:1171-1179.

12. Deignan, T., et al. 2002. Decrease in hepatic CD56(+) T cells and V alpha 24(+) natural killer T cells in chronic hepatitis $\mathrm{C}$ viral infection. J. Hepatol. 37:101-108.

13. Durante-Mangoni, E., et al. 2004. Hepatic CD1d expression in hepatitis $\mathrm{C}$ virus infection and recognition by resident proinflammatory CD1d-reactive T cells. J. Immunol. 173:2159-2166.

14. Kawarabayashi, N., et al. 2000. Decrease of CD56(+)T cells and natural killer cells in cirrhotic livers with hepatitis $\mathrm{C}$ may be involved in their susceptibility to hepatocellular carcinoma. Hepatology. 32:962-969.

15. Rosen, H.R., et al. 2008. Pretransplantation CD56(+) innate lymphocyte populations associated with severity of hepatitis $C$ virus recurrence. Liver Transpl. 14:31-40.

16. Doherty, D.G., et al. 1999. The human liver contains multiple populations of NK cells, T cells, and $\mathrm{CD} 3+\mathrm{CD} 56+$ natural $\mathrm{T}$ cells with distinct cytotoxic activities and Th1, Th2, and Th0 cytokine secretion patterns. J. Immunol. 163:2314-2321.

17. Mazzaferro, V., et al. 1996. Liver transplantation for the treatment of small hepatocellular carcinomas in patients with cirrhosis. N. Engl. J. Med. 334:693-699. 18. Mercer, D.F., et al. 2001. Hepatitis C virus replication in mice with chimeric human livers. Nat. Med. 7:927-933.

19. Kneteman, N.M., et al. 2006. Anti-HCV therapies in chimeric scid-Alb/uPA mice parallel outcomes in human clinical application. Hepatology. 43:1346-1353.

20. Tateno, C., et al. 2004. Near completely humanized liver in mice shows human-type metabolic responses to drugs. Am. J. Pathol. 165:901-912.

21. Tseng, C.T., and Klimpel, G.R. 2002. Binding of the hepatitis $\mathrm{C}$ virus envelope protein E2 to CD81 inhibits natural killer cell functions. J. Exp. Med. 195:43-49.

22. Crotta, S., et al. 2002. Inhibition of natural killer cells through engagement of CD81 by the major hepatitis C virus envelope protein. J. Exp. Med. 195:35-41.

23. Wang, S.H., et al. 2008. Natural killer cells suppress full cycle $\mathrm{HCV}$ infection of human hepatocytes. J. Viral Hepat. 15:855-864.

24. Kanto, T., and Hayashi, N. 2007. Innate immunity in hepatitis $C$ virus infection: Interplay among dendritic cells, natural killer cells and natural killer T cells. Hepatol. Res. 37(Suppl. 3):S319-S326.

25. Yamagiwa, S., et al. 2008. Sustained response to interferon-alpha plus ribavirin therapy for chronic hepatitis C is closely associated with increased dynamism of intrahepatic natural killer and natural killer T cells. Hepatol. Res. 38:664-672.

26. Van Wauwe, J.P., De Mey, J.R., and Goossens, J.G. 1980. OKT3: a monoclonal anti-human T lymphocyte antibody with potent mitogenic properties. J. Immunol. 124:2708-2713.

27. Chang, T.W., Kung, P.C., Gingras, S.P., and Goldstein, G. 1981. Does OKT3 monoclonal antibody react with an antigen-recognition structure on human T cells? Proc. Natl. Acad. Sci. U. S. A 78:1805-1808.

28. Chatenoud, L., et al. 1990. In vivo cell activation following OKT3 administration. Systemic cytokine release and modulation by corticosteroids. Transplantation. 49:697-702.

29. Soza, A., et al. 2005. Pilot study of interferon gamma for chronic hepatitis C. J. Hepatol. 43:67-71.

30. Jo, J., et al. 2009. Analysis of CD8+ T-cell-mediated inhibition of hepatitis $\mathrm{C}$ virus replication using a novel immunological model. Gastroenterology. 136:1391-1401.

31. Penna, A., et al. 2007. Dysfunction and functional restoration of $\mathrm{HCV}$-specific CD8 responses in chronic hepatitis $\mathrm{C}$ virus infection. Hepatology. 45:588-601.

32. Ohdan, H., et al. 2003. Intraoperative near-infrared spectroscopy for evaluating hepatic venous outflow in living-donor right lobe liver. Transplantation. 76:791-797.

33. Tanaka, Y., Ohdan, H., Onoe, T., and Asahara, T, 2004. Multiparameter flow cytometric approach for simultaneous evaluation of proliferation and cytokine-secreting activity in $\mathrm{T}$ cells responding to allo-stimulation. Immunol. Invest. 33:309-324.

34. Guo, J.T., Bichko, V.V., and Seeger, C. 2001. Effect of alpha interferon on the hepatitis $\mathrm{C}$ virus replicon. J. Virol. 75:8516-8523.

35. Tanabe, Y., et al. 2004. Synergistic inhibition of intracellular hepatitis $\mathrm{C}$ virus replication by combination of ribavirin and interferon- alpha. J. Infect. Dis. 189:1129-1139.

36. Yokota, T., et al. 2003. Inhibition of intracellular hepatitis $C$ virus replication by synthetic and vector-derived small interfering RNAs. EMBO Rep. 4:602-608.

37. Tsuge, M., et al. 2005. Infection of human hepatocyte chimeric mouse with genetically engineered hepatitis B virus. Hepatology. 42:1046-1054.

38. Hiraga, N., et al. 2007. Infection of human hepatocyte chimeric mouse with genetically engineered hepatitis $C$ virus and its susceptibility to interferon. FEBS Lett. 581:1983-1987.

39. Kimura, T., et al. 2008. Establishment of an infectious genotype $1 \mathrm{~b}$ hepatitis $\mathrm{C}$ virus clone in human hepatocyte chimeric mice. J. Gen. Virol. 89:2108-2113. 